OAI-PMH: http://www.indteca.com/ojs/index.php/Revista Scientific/oai

Artículo Original / Original Article

\title{
La política pública de Educación Superior en el Ecuador: Un ejercicio contextualizado de análisis crítico
}

\author{
Autores: Carlos Andrés Ortega Vega \\ Secretaría de Educación Superior, Ciencia, Tecnología e Innovación, SENESCYT \\ cortega@senescyt.gob.ec \\ Quito, Ecuador \\ https://orcid.org/0000-0001-6516-7758 \\ Christian Virgilio Guerrero Salazar \\ Asesoría y Capacitación Pedagógica, ASCAPED \\ chguerrero.ascaped@gmail.com \\ Guayaquil, Ecuador \\ https://orcid.org/0000-0003-3108-1846
}

\section{Resumen}

El presente análisis, establece los antecedentes históricos de la política pública en el Ecuador, en lo referente a la Educación superior, permitiendo visualizar un contexto que permita comprender la realidad acerca de la eficacia en las propuestas vinculadas con el mejoramiento educativo y la calidad de sus procesos, tanto de planificación como de diseño, implementación y evaluación. La metodología utilizada es la del análisis documental, por medio de la recopilación de los datos obtenidos acerca de una política pública específica, tomada como ejemplo, para establecer comparativamente la eficiencia de su aplicación en el contexto real, contrastándolo con la bibliografía hegemónica existente acerca del tema y el criterio de expertos. El estudio se basa en tres ejes teóricos que son: el ciclo, los actores y las reglas de juego, para luego poder valorar los resultados obtenidos por la política pública en cuestión; generando al final un conjunto de conclusiones y recomendaciones emanadas del análisis y la experiencia en equipos de gestión pública.

Palabras clave: política pública; educación superior; gobernabilidad; gestión pública.

Código de clasificación internacional: 5902.07 - Política educativa.

\section{Cómo citar este artículo:}

Ortega, C., \& Guerrero, C. (2021). La política pública de Educación Superior en el Ecuador: Un ejercicio contextualizado de análisis crítico. Revista Scientific, 6(20), 19-40, e-ISSN: 2542-2987.

Recuperado de: https://doi.org/10.29394/Scientific.issn.2542-2987.2021.6.20.1.19-40

Fecha de Recepción: 16-01-2021
Fecha de Aceptación: 24-03-2021
Fecha de Publicación: 05-05-2021 
OAl-PMH: http://www.indteca.com/ojs/index.php/Revista Scientific/oai

Artículo Original / Original Article

\title{
The public policy of Higher Education in Ecuador: A contextualized exercise of critical analysis
}

\begin{abstract}
This analysis establishes the historical antecedents of public policy in Ecuador, in relation to higher education, allowing to visualize a context that allows understanding the reality about the effectiveness of the proposals related to educational improvement and the quality of its processes, both planning and design, implementation and evaluation. The methodology used is that of documentary analysis, through the compilation of data obtained about a specific public policy, taken as an example, to comparatively establish the efficiency of its application in the real context, contrasting it with the existing hegemonic bibliography about the subject and expert judgment. The study is based on three theoretical axes which are: the cycle, the actors and the rules of the game, in order to then be able to assess the results obtained by the public policy in question; generating at the end a set of conclusions and recommendations emanating from the analysis and experience in public management teams.
\end{abstract}

Keywords: public politics; higher education; governance; public management. International classification code: 5902.07 - Educational policy.

How to cite this article:

Ortega, C., \& Guerrero, C. (2021). The public policy of Higher Education in Ecuador: A contextualized exercise of critical analysis. Revista Scientific, 6(20), 19-40, e-ISSN: 2542-2987. Recovered from: https://doi.org/10.29394/Scientific.issn.2542-2987.2021.6.20.1.19-40

Date Received: 16-01-2021
Date Acceptance: 24-03-2021
Date Publication: 05-05-2021 


\section{Introducción}

En el contexto latinoamericano, el diseño y aplicación de políticas públicas, históricamente ha estado ligado a la tendencia política de los gobiernos de turno, presentando comúnmente una grieta muy amplia entre la verdadera necesidad social y los intereses particulares de quienes ejercen el poder.

La eficacia de las políticas públicas aplicadas en América Latina hasta la primera década del siglo XXI, según el informe del Banco Interamericano de Desarrollo BID, no fueron exitosas en su gran mayoría, surgiendo, conforme a Stein, Tommasi, Echebarría, Lora y Payne (2006a): existen "[...] dudas con respecto a la eficiencia de las mismas y un debate sobre el rumbo que debería tomar la política económica y social a fin de alcanzar la elusiva meta del crecimiento sostenible [...]" (pág. 5). Esto causó una gran incertidumbre sobre el futuro, provocando un análisis profundo de las posibles causas que pudieran estar incidiendo tanto en las propuestas, el diseño o la aplicación de estas, para su fracaso.

En este ámbito, es importante acotar, que el fracaso de una política pública, no estriba solamente en la poca efectividad de sus resultados a corto o largo plazo, sino más bien en un grupo de factores interrelacionados, que pueden ir desde una deficiente delimitación del problema, la falta de probidad científica en las teorías que sustentan el accionar frente al problema, la falta de liderazgo de los actores que dirigen la iniciativa, el inadecuado seguimiento de las acciones propuestas, la falta de proyección respecto a los alcances de los proyectos diseñados, hasta inclusive la falta de ética en el manejo de los mismos.

Mencionando a Scartascini, Spiller, Stein y Tommasi (2011a): en el contexto histórico de países como el Ecuador, la inestabilidad social y política vivida hasta la primera década del siglo XXI, impidió un manejo eficiente de las políticas públicas, ya sea por la falta de probidad de los líderes, como por 
el exceso de actores cuyo consentimiento era necesario, pero actuaban de forma poco cooperativa, produciendo políticas sociales y económicas de baja calidad.

De acuerdo con Stein, Tommasi, Echebarría, Lora y Payne (2006b): en estos países con sistemas políticos fragmentados, se torna más difícil la gobernabilidad, dependiendo mucho de que el presidente tenga mayoría legislativa, o en su defecto una gran capacidad para formar o mantener coaliciones que beneficien al Estado en la aprobación y manejo de la política para el bien común.

Los cambios sustanciales en la visibilidad de las políticas públicas y su manejo en Ecuador, se dieron con la llegada de la llamada revolución ciudadana al poder, y la nueva constituyente a partir del año 2006. Estos cambios propiciaron una mejor estructura en los análisis, aunque no necesariamente en los efectos finales de su aplicación, sobre todo en lo referente a la libertad de acuerdos y posturas sobre su importancia.

Esto se debió principalmente a una estrategia no necesariamente correcta desde el punto de vista ético, ya que, aunque Rafael Correa no había llegado al poder, teniendo peso en el Congreso, logró reconfigurar una mayoría que aprobara la consulta popular para que se instaure la Asamblea Constituyente, destituyendo a 57 legisladores que habían firmado en contra, reemplazándolos por gente afín a su proyecto, incluso como mencionan Basabe-Serrano, Pachano y Mejía (2010): "a pesar de que el Tribunal Constitucional había declarado que la decisión del TSE era violatoria a la Carta Política" (pág. 77).

Otros dos factores que favorecieron el panorama en el manejo de las políticas públicas, fueron la participación ciudadana y la bonanza petrolera que vivió el gobierno del presidente Rafael Correa desde el inicio de su gestión, afianzada sin lugar a dudas por la dolarización. Cabe recalcar que, en lo referente a la participación ciudadana, aunque fue evidente su presencia a 
través de diferentes organizaciones, el ejecutivo mantuvo siempre una poderosa influencia sobre las decisiones de dichos colectivos determinando así un control casi total de los apoyos necesarios para la aprobación de leyes. Esto produjo, a decir de Scartascini, Spiller, Stein y Tommasi (2011b): que el nuevo proyecto constitucional fortaleciera aún más el poder relativo y absoluto de los actores decisivos, neutralizando el poder de otros actores que podían vetar las decisiones e incorporando a nuevos actores dirimentes afines a la línea planteada por el ejecutivo.

Bajo estos nuevos lineamientos de acción política, los cuales se han mantenido hasta la actualidad, con ciertas variaciones, se creó el Plan Nacional de Desarrollo (PND) en el año 2007, que, de acuerdo a la Constitución de la República del Ecuador (2008a): es "el instrumento al que se sujetarán las políticas, programas y proyectos públicos [...]" (art. 280); el cual contiene el compendio de las políticas públicas proyectadas a un plazo general de 4 años, en los distintos aspectos sociales, económicos y educativos.

La última reformulación de este plan se produjo en el año 2017, ya en el gobierno de Lenin Moreno con un alcance de cumplimiento hasta el año 2021. El documento está organizado en tres ejes principales, cada uno con tres objetivos y un total de 81 políticas públicas con sus respectivas metas en función del cumplimiento de los objetivos planteados. Con base en esta estructura diseñada y con base en lo descrito por Senplades (2017a): la política general escogida para su análisis en este trabajo es "garantizar el derecho a la salud, la educación y al cuidado integral durante el ciclo de vida, bajo criterios de accesibilidad, calidad y pertinencia territorial y cultural" (pág. 58); bajo la especificidad de una de sus metas puntuales que es "incrementar del $27,81 \%$ al $31,21 \%$ la tasa bruta de matrícula en educación superior en Universidades y Escuelas Politécnicas a 2021" (pág. 59). 


\section{Metodología}

Por su naturaleza de análisis, el estudio se considera cualitativo, con un alcance descriptivo, ya que busca determinar la eficacia de la aplicación de políticas públicas en el contexto real de gobernabilidad establecido en el Ecuador, debido a los cambios políticos generados a partir del año 2006, en contraste con lo actuado en escenarios anteriores.

Debido a las fuentes con que se diseña, se considera documental, ya que, al seleccionar una política específica, se realiza un análisis comparativo para determinar sus características teóricas, sobre la base de la bibliografía más relevante existente acerca de la materia, cuyos autores proponen los fundamentos esenciales para el reconocimiento de los estándares de calidad que deben cumplir las políticas públicas eficientes.

El análisis se establece bajo tres ejes teóricos que son: el ciclo o proceso, los actores involucrados y las reglas del juego, para luego poder establecer los resultados obtenidos en su aplicación y determinar el nivel de eficacia, así como los puntos a mejorar.

\subsection{Ciclo o proceso de política pública}

Es necesario recordar a Tamayo (1997), quien expresa que el proceso o ciclo de construcción de las políticas públicas comprende las siguientes fases: "1. Identificación y definición del problema; 2. Formulación de las alternativas de solución; 3. Adopción de una alternativa; 4. Implantación de la alternativa seleccionada; 5. Evaluación de los resultados obtenidos" (pág. 281).

La política escogida, tiene como objetivo propuesto por la Senplades (2017b): el "garantizar una vida digna con iguales oportunidades para todas las personas" (pág. 53); que en conjunto con su meta específica nos indica que su formulación está ligada a la problemática general del acceso a la educación, en este caso limitándola particularmente al nivel superior, 
correspondiente en el Ecuador a las llamadas Instituciones de Educación Superior (IES) que de acuerdo a la Ley Orgánica de Educación Superior (LOES, 2018a), son:

a). Las universidades, escuelas politécnicas públicas y particulares, debidamente evaluadas y acreditadas, conforme la presente Ley; b). Los institutos superiores técnicos, tecnológicos, pedagógicos y de artes, tanto públicos como particulares debidamente evaluados y acreditados, conforme la presente Ley; y, c). Los conservatorios superiores, tanto públicos como particulares, debidamente evaluados y acreditados, conforme la presente Ley (art. 14).

Esta estructura responde a las necesidades específicas del país, de manera que entra en concordancia con los niveles de formación que establece la misma Ley Orgánica de Educación Superior (LOES, 2018b): en su artículo 118, el cual se divide en un tercer nivel, que puede ser a la vez de tipo técnicotecnológico o de grado; y un cuarto nivel o posgrado, que a su vez también puede ser de tipo tecnológico (especialistas y maestrías tecnológicas) o académico (maestrías y PhD.). Para este análisis, nos centraremos en el acceso únicamente al tercer nivel de educación superior desde el bachillerato.

Esto nos permite identificar el problema principal como la falta de acceso a la educación superior de los jóvenes ecuatorianos al terminar la educación media, ya sea debido a problemas socio económicos que los obligan a insertarse en el mundo laboral, como a la falta de interés por la obtención de un título superior.

En el Ecuador, a partir del año 2010, se creó la Secretaría de Educación Superior, Ciencia, Tecnología e Innovación (SENESCYT), a través de la fusión de varias entidades anteriores, entre ellas el llamado Consejo Nacional de Educación Superior (CONESUP), cuyas funciones en teoría eran similares, pero su administración y funcionamiento estuvo plagado de irregularidades, así como también de limitaciones en el momento de establecer prioridades 
OAI-PMH: http://www.indteca.com/ojs/index.php/Revista Scientific/oai

\section{Artículo Original / Original Article}

para el análisis técnico de las políticas y proyectos a su cargo. En este mismo orden de ideas, Rojas (2011), denuncia que:

En la práctica, la falta de aprobación oportuna de varios reglamentos por parte del CONESUP [...] y la falta de implementación de mecanismos de seguimiento y control permanente a las actividades académicas universitarias, permitió a un buen número de universidades y escuelas politécnicas la proliferación indiscriminada de los programas de posgrado y la creación de numerosas carreras de grado que respondían más bien a una demanda clientelar y no a una verdadera necesidad social, convirtiendo de esta manera a la educación superior en un "buen negocio", actitud que afectó significativamente su calidad (pág. 60).

En este contexto, eran urgentes los cambios, que permitieran superar muchos de los conflictos y problemas que se habían arraigado en la Educación Superior, desacreditándola y mermando cada vez más su calidad. Esto fue posible por medio de propuestas no solo de forma, sino estructurales y de base como las que se sintetizan brevemente en la tabla 1.

Tabla 1. Comparativa CONESUP-SENESCYT.

\begin{tabular}{|c|c|c|}
\hline Aspecto & CONESUP & SENESCYT \\
\hline Acceso & $\begin{array}{l}\text { Respecto al acceso, Ramírez (2016a): } \\
\text { respecto al acceso, se utilizaron } \\
\text { "políticas heterogéneas entre las } \\
\text { universidades y escuelas politécnicas, } \\
\text { entre las de carácter público y privado, } \\
\text { y entre las distintas carreras de las } \\
\text { mismas instituciones de educación } \\
\text { superior [...]" (pág. 140). }\end{array}$ & $\begin{array}{l}\text { Se estableció el Sistema } \\
\text { Nacional de Admisión y } \\
\text { Nivelación SNNA, proyecto de } \\
\text { SENESCYT, que estableció de } \\
\text { manera obligatoria, los cursos } \\
\text { de nivelación, que pueden ser } \\
\text { descartados si se aprueba un } \\
\text { examen de ingreso. }\end{array}$ \\
\hline $\begin{array}{l}\text { Examen/ } \\
\text { Admisión }\end{array}$ & $\begin{array}{l}\text { Se aplicaban un sinnúmero de } \\
\text { variantes como formas de ingreso a } \\
\text { las universidades, la gran mayoría } \\
\text { decididas por las mismas } \\
\text { instituciones, y sin apego real a la ley, } \\
\text { lo cual impidió, acorde con Ramírez } \\
\text { (2016b): "[...] la aplicación de las } \\
\text { políticas de admisión y nivelación a la } \\
\text { educación superior en el país [...]" } \\
\text { (pág. 140). }\end{array}$ & $\begin{array}{l}\text { Se creó el Examen Nacional de } \\
\text { Educación Superior (ENES), } \\
\text { que como indica Ramírez } \\
\text { (2016c): se convirtió en el } \\
\text { "inicio de la postulación a los } \\
\text { cupos ofertados por las } \\
\text { universidades e institutos de } \\
\text { educación superior" (pág. 144); } \\
\text { herramienta estandarizada que } \\
\text { garantiza una más justa } \\
\text { "asignación de cupos en } \\
\text { función al mérito de cada } \\
\text { estudiante" (pág. 145). }\end{array}$ \\
\hline
\end{tabular}


Instituto Internacional de Investigación y Desarrollo Tecnológico Educativo INDTEC, C.A.

DOI: https://doi.org/10.29394/Scientific.issn.2542-2987.2021.6.20.1.19-40

OAI-PMH: http://www.indteca.com/ojs/index.php/Revista Scientific/oai

Artículo Original / Original Article

\begin{tabular}{|c|c|c|}
\hline Gratuidad & $\begin{array}{l}\text { Cobro por parte de las IES privadas en } \\
\text { función de sus políticas particulares, } \\
\text { mientras que las IES públicas también } \\
\text { cobraban, pero solo la matrícula cada } \\
\text { año. Poco acceso a puntos remotos } \\
\text { del país, de manera que los } \\
\text { estudiantes del interior de las } \\
\text { provincias debían trasladarse a las } \\
\text { grandes ciudades e incurrir en gastos } \\
\text { adicionales. }\end{array}$ & $\begin{array}{l}\text { Con la reforma a la } \\
\text { Constitución de la Republica } \\
\text { del Ecuador (2008): se } \\
\text { garantiza que la enseñanza } \\
\text { universitaria y politécnica de } \\
\text { carácter público sea "gratuita } \\
\text { hasta el tercer nivel" (art. 28); } \\
\text { además que, si bien se permitió } \\
\text { que las instituciones privadas } \\
\text { sigan cobrando aranceles se } \\
\text { les exigió que diseñaran } \\
\text { mecanismos para la equidad e } \\
\text { integración social tales como } \\
\text { becas, créditos, cuotas de } \\
\text { ingreso, entre otros. }\end{array}$ \\
\hline Matr & $\begin{array}{l}\text { Conforme a lo descrito por Ramírez y } \\
\text { Minteguiaga (2010): "mientras que en } \\
\text { el año } 1995 \text { la matrícula del } 20 \text { por } \\
\text { ciento más rico de la población era } 2.5 \\
\text { veces a la del } 20 \text { por ciento más pobre, } \\
\text { trece años después la relación era } 6 \\
\text { veces más" (pág. 139); lo cual } \\
\text { evidenciaba el alto nivel de } \\
\text { desigualdad respecto a las } \\
\text { posibilidades de acceso de la } \\
\text { población. }\end{array}$ & $\begin{array}{l}\text { Como declara la Secretaría de } \\
\text { Educación Superior, Ciencia, } \\
\text { Tecnología e Innovación } \\
\text { (SENESCYT, 2020): se } \\
\text { produjo un cierre de la brecha } \\
\text { de equidad ya que para el } \\
\text { 2018, el número de estudiantes } \\
\text { matriculados en IES privados } \\
\text { tanto autofinanciados como } \\
\text { cofinanciados fue de } 254.017 \text {, } \\
\text { mientras que el de } \\
\text { matriculados en IES de } \\
\text { carácter público fue de } \\
378.524 \text {. }\end{array}$ \\
\hline Infor & $\begin{array}{l}\text { Existía demasiada desinformación, } \\
\text { porque aparte del poco interés que a } \\
\text { veces se mostraba, las Instituciones } \\
\text { de Educación superior, no contaban } \\
\text { aún en su gran mayoría con } \\
\text { plataformas de información o páginas } \\
\text { web como las de la actualidad, }\end{array}$ & $\begin{array}{l}\text { Se creó un sistema integrado } \\
\text { de acceso a la información de } \\
\text { las IES tanto públicas como } \\
\text { privadas que permitieron el } \\
\text { reconocimiento de su aval, } \\
\text { categoría, así mismo como los } \\
\text { enlaces de acceso a sus } \\
\text { ofertas académicas. }\end{array}$ \\
\hline
\end{tabular}

Fuente: Los Autores (2021).

Actualmente la SENESCYT, tiene a su cargo la rectoría de la política pública, así como la formulación técnica de los mecanismos de diseño, implementación y evaluación de los proyectos asociados a las metas que involucran como en este caso a la educación superior. Esta secretaría, por medio de su proyecto llamado Sistema Nacional de nivelación y admisión (SNNA), ha sido la encargada de plantear alternativas de solución a la 
Instituto Internacional de Investigación y Desarrollo Tecnológico Educativo INDTEC, C.A.

DOI: https://doi.org/10.29394/Scientific.issn.2542-2987.2021.6.20.1.19-40

OAI-PMH: http://www.indteca.com/ojs/index.php/Revista Scientific/oai

Artículo Original / Original Article

problemática hallada, entre las que se pueden indicar:

- Elaborar un plan de becas que permitan el acceso a la educación superior para estudiantes de bajos recursos económicos que egresaren de los colegios públicos del país.

- Otorgar ayudas económicas exclusivamente con fines académicos, a: estudiantes, docentes, investigadores y profesionales, para el desarrollo de proyectos de investigación y perfeccionamiento profesional.

- Incrementar la capacidad instalada de las instituciones de educación superior: planta docente, infraestructura y equipamiento.

- Diversificar la oferta por área de conocimiento con pertinencia y calidad para atender las necesidades sociales y productivas del país.

La implementación de las políticas públicas se definió a través de diferentes procesos como proyectos de inversión nombrados como Fortalecimiento de la educación superior universitaria, técnica y tecnológica con criterios de inclusión, calidad y pertinencia, el Programa de Reforzamiento Académico (PRA), los Grupos de alto Rendimiento (GAR), así como convenios interinstitucionales que permiten el desarrollo de los objetivos referentes a la meta propuesta por medio de convenios Interinstitucionales para el levantamiento de datos a través del Instituto Nacional de Estadística y Censos (INEC), inversión en becas de investigación y profesionalización a cargo del Instituto de Fomento al Talento Humano (IFTH), entre otros.

La evaluación de los resultados de las políticas aplicadas, se establecen a través de un conjunto de indicadores de gestión organizados en matrices de evaluación apropiadas y concordantes con los procesos analizados. Este análisis de Whittingham (2005), citado por Bravo (2018), se realiza en función de la:

[...] Definición de cuatro variables principales (Participación, 
Equidad, Rendición de cuentas, Eficiencia), siete variables secundarias o subvariables (Capacidad de respuesta, Innovación administrativa y gerencial, Interacción Estadociudadanos-ONGs, Administración descentralizada, Creación de redes, Desarrollo del recurso humano) y, sus indicadores (pág. 28).

Entre los indicadores asociados al cumplimiento de la meta propuesta tenemos: 1. Estimación de tendencia permanente (antes de la pandemia); 2. Cálculo de indicador real; 3. Evolución de inscritos en procesos de acceso a la educación superior; y 4. Evaluación multisectorial de los efectos e impactos del COVID-19 en Educación Superior realizada por la Organización de las Naciones Unidas para la Educación, la Ciencia y la Cultura (UNESCO).

\subsection{Actores involucrados}

En el desarrollo de cualquier acción que involucre transformaciones o cambios en las variables que sustentan al fenómeno estudiado, existen individuos o instituciones que proporcionan los medios intelectuales y físicos para que sea posible dicha acción. En términos de política pública, un actor es la persona o grupo de personas que cumplen un rol determinado en la determinación, diseño o implementación de las acciones propuestas para su cumplimiento.

Estos actores, se involucran en el proceso de los proyectos que llevan a cabo el cumplimiento de las políticas, de tal manera que tarde o temprano se interrelacionan con el objetivo propuesto con base en sus propias características sociales y defendiendo una postura inherente acerca de la situación. Para Pérez (2016a): se requiere "analizar los actores implica reconocer los valores, creencias y modelos mentales que dan lugar a definiciones específicas de un problema de política y al perfilamiento de estrategias y tácticas que definen su margen de acción" (pág. 256).

De acuerdo a Jordana (1995): se debe "[...] entender que las decisiones 
políticas surgen en redes de actores, públicos y privados, que interactúan en ámbitos sectoriales o plurisectoriales; y en niveles regionales, nacionales 0 internacionales" (pág. 7). Por otro lado, en los procesos de las políticas públicas, siempre existirán actores que se encuentran formalmente involucrados en el proceso, como otros informales que, aunque no son afectados directamente pueden verse beneficiados de los resultados obtenidos.

Con base en estas apreciaciones, y en correspondencia con la clasificación específica de Pérez (2016b), podemos establecer cinco categorías de actores que se evidencian en el análisis de nuestra política escogida:

Los actores políticos; son de carácter público formal y en cierto modo son los que toman la decisión para la formulación de la política sobre la base del problema identificado, se encargan de su promulgación y aceptación entre los diferentes estamentos estatales. En nuestro caso estos corresponderían al poder ejecutivo (presidente Lenin Moreno), el Consejo nacional de Planificación que aprobó el PND y al poder legislativo (Asamblea Nacional) en su calidad de veedor del cumplimiento de las políticas públicas.

Los actores burocráticos; también son de carácter público formal y engloba a los diferentes especialistas (investigadores, juristas y revisores), técnicos y personal administrativo involucrado en el estudio de los problemas, el diseño, redacción, diagramación y edición final de los documentos oficiales que contienen el conjunto de políticas a desarrollarse. En esta categoría se encuentra la Secretaría Nacional de Planificación y Desarrollo y su personal involucrado en el desarrollo del PND, así como la SENESCYT en su papel específico de cubrir el análisis metodológico y ejecutar la norma técnica para la meta propuesta respecto a la tasa de matriculación en la Educación Superior.

La población objetivo; pueden ser de carácter público o privado, pero 
definitivamente se consideran formales, ya que son los individuos de la sociedad que forman parte de la problemática, sea como causantes o afectados. En nuestro caso podríamos considerar en esta categoría, tanto a la población estudiantil egresada de la educación media, fluctuante entre las edades de 18 a 24 años, como al conjunto de instituciones de educación superior, que se ven afectadas de forma directa por la tasa creciente de deserción y los porcentajes elevados de estudiantes que descartan los estudios superiores.

Los beneficiarios finales; en este ámbito debemos aclarar que no solamente se consideran a quienes resultan beneficiados de forma directa, sino que en ocasiones también se amplía a otros grupos poblacionales que lo hacen de forma indirecta. En el caso que nos compete podríamos indicar que, además de los grupos nombrados como población objetivo, se benefician también las familias subyacentes, ya que, al mejorar la tasa de matriculación, a largo plazo y dependiendo de la culminación de las carreras, tendríamos un beneficio en la obtención de puestos laborales mejor remunerados.

Por último, están los actores terciarios; son principalmente de carácter privado e informales. Son principalmente aquellos actores con intereses generales, especiales o intelectuales expertos, que debido a sus recursos cognitivos o académicos resultan en cierto modo involucrados indirectamente y a la vez beneficiados por el conjunto de necesidades que generan en diversos ámbitos las acciones propuestas.

En nuestro caso podríamos nombrar a equipos de asesores llamados a concurso para proyectos de diseño metodológico de evaluación de procesos, así como las instituciones privadas que se dedican a la preparación de estudiantes para el ingreso a la Universidad.

\subsection{Las reglas de juego}

Como se dice comúnmente en los deportes, no basta con ser un buen 
jugador, se deben conocer las reglas del juego. $Y$ es que, en ocasiones, este conocimiento te brinda oportunidades de triunfar, más allá de la experticia; esto no significa que se desdeñe el conocimiento ni la experiencia, sino por el contrario, el conocer las reglas del juego potencia en gran medida aquello ganado con el estudio y la preparación.

En este sentido, Ostrom (2015): formula que, cuando se usa en su sentido regulatorio, las reglas se refieren a algo "establecido por una autoridad (una legislación, un juez, magistrado, consejo directivo, rector universitario, padre o madre) y que obliga a determinadas personas (o bien les prohíbe o les permite)" (pág. 60); en el campo de las políticas públicas, existen variadas perspectivas que de una u otra manera, definen los tipos de reglas, como parte de un escenario completo que contiene diversos elementos interrelacionados, como el modelo que se presenta en la tabla 2.

Tabla 2. El juego de las políticas.

\begin{tabular}{|c|l|l|}
\hline Categoría & \multicolumn{1}{|c|}{ Concepto } & \multicolumn{1}{c|}{ Autor } \\
\hline Reglas & Regulación (+ normas + estrategias) & D. North, E. Ostrom \\
\hline Jugadores & Organizaciones, actores & D. North, E. Ostrom \\
\hline Terreno & Arena de acción & E. Ostrom \\
\hline Juego & La política importa & D. Acemoglu \\
\hline
\end{tabular}

Fuente: Eslava (2011).

En atención a Subirats, Knoepfel, Larrue y Varone (2008a), el analista de una política pública puede diferenciar tres niveles generales de organización de las reglas institucionales:

Las reglas constitucionales; corresponden al régimen democrático, y comprenden los principios jurídicos y derechos establecidos en la Constitución. Particularmente en las políticas públicas de nuestro país, estas reglas están determinadas por los principios básicos de los $\mathrm{DDHH}$, así como en los artículos referidos de la Constitución de la República del Ecuador (2008b): para el desarrollo del PND, específicamente el 280 y 293. 
Las regulaciones de organizaciones públicas; que corresponden a la organización y regulación de los entes públicos encargados de gestionar las políticas de acción. En este apartado tenemos principalmente a los estatutos y normas de acción administrativa de la Senplades, Senescyt, así como el mismo PND que es el conjunto de normas, criterios e indicadores que determinan el accionar de las políticas públicas en el Ecuador.

Los acuerdos político-administrativos; constituyen a tenor de Subirats, Knoepfel, Larrue y Varone (2008b): "el contenido específico de la acción pública, propio de cada política” (pág. 105). Para el caso particular de la política estudiada, se establecen como acuerdos específicos algunos cuerpos normativos de accionar para la Senplades, como la Guía para la formulación de políticas públicas sectoriales, Guía Metodológica de Planificación Institucional, la Propuesta de Lineamientos Generales para la Planificación Territorial Descentralizada.

Por otro lado, se encuentra la SENESCYT, como institución responsable de la fuente de información para el desarrollo de las notas técnicas de metas del PND, a través de sus Registros administrativos; así como también el INEC por medio de las Proyecciones de población.

Por último, están los acuerdos interinstitucionales que se establecen con la finalidad de fortalecer las acciones de gestión administrativa y económica en pro de las políticas públicas. Entre otros, tenemos el acuerdo Senplades-HUB norte con la finalidad de mejorar la investigación, innovación, emprendimiento y la aplicación de metodologías de la planificación para el desarrollo de la zona norte del país; así como el convenio para la implementación del programa de becas de grado y posgrado entre la SENESCYT, el Ministerio de Salud Pública y el Instituto de Fomento al Talento Humano. 


\section{Resultados}

Sobre la base de los indicadores detallados en la sección de ciclo o proceso de la política pública de este mismo escrito, podemos establecer los resultados parciales obtenidos por la política escogida en función de la meta de aumentar del $27,81 \%$ al $31,21 \%$ la tasa bruta de matrícula en educación superior hasta el año 2021 como los siguientes:

- Evolución de la matrícula en tercer nivel del período 2015-2018 con información del Sistema Integral de Información de Educación Superior (SIIES), mediante el cual se evidencia el crecimiento tendencial sin afectación del COVID-19, expuesto en la tabla 3.

- Inscritos en el proceso de acceso a la educación superior en el segundo semestre del año 2020, en donde se evidencia una disminución de los inscritos en las IES públicas.

Tabla 3. Registro de matrícula de Universidades y Escuelas Politécnicas.

\begin{tabular}{|c|c|c|c|c|}
\hline Nivel de Formación & $\mathbf{2 0 1 5}$ & $\mathbf{2 0 1 6}$ & $\mathbf{2 0 1 7}$ & $\mathbf{2 0 1 8}$ \\
\hline Tercer Nivel o Pregrado & 540.282 & 540.057 & 564.773 & 605.185 \\
\hline
\end{tabular}

Fuente: SENESCYT (2020).

Entre los años 2019 y 2020, la pandemia del COVID-19, modificó la evaluación de los proyectos y políticas específicas aplicadas a la meta de incrementar la tasa bruta de matrícula en educación superior de Universidades y Escuelas Politécnicas, para lo cual se habían definido mecanismos técnicos del cálculo requerido, por medio de un análisis tendencial con base en el comportamiento histórico de la tasa bruta, comparándolo con el obtenido en el momento de la evaluación.

Esta metodología partió de la definición de la fórmula de tasa bruta, como el cociente entre la población matriculada en tercer nivel en establecimientos de educación superior, y el número de personas en edades 
entre los 18 y 24 años, como denotan las proyecciones proporcionadas por el INEC. Este cálculo permitió estimar una involución en la tendencia de incremento de $3.27 \%$ en 2020, a $2.93 \%$ en 2021 que, aunque era de esperarse debido a la crisis económica y social por la pandemia, trastocó los planes iniciales hasta el punto de tener que modificar la meta inicial en el porcentaje propuesto a alcanzarse.

\section{Conclusiones}

A manera de conclusión, podemos anotar en forma general que el manejo de las políticas públicas, sobre todo en lo referente a la Educación superior, experimentó a partir del año 2010 un potente resurgimiento de la mano con la implementación de procesos consensuados y organizados, que permitieron un análisis más técnico y académico de las políticas, generadas en función de un esquema de investigación social y científicamente riguroso.

Al mejorar la generación de políticas más acordes con las problemáticas sociales, estas pudieron ser aplicadas con la base de equipos técnicos bien estructurados -actores- y delimitados que cumplieran con sus funciones de una manera más eficiente.

Las políticas públicas en Educación superior, abarcan un gran campo de acción que, si bien están en concordancia con el PND y buscan generar soluciones a las principales problemáticas sociales de acceso y permanencia en la educación, es justamente ese abarcar lo que provoca que muchas de esas políticas se diluyan en los procesos y no se logren alcanzar las metas propuestas.

En cuanto a la política pública analizada, podemos concluir que su manejo técnico fue el adecuado, al menos hasta antes de la pandemia, cuando se pudo visualizar en los reportes de la SENESCYT, una tendencia en aumento del 2017 al 2018 de casi el 7\% de la tasa de variación ajustada.

Las variables no previstas debido a la pandemia debilitaron la tendencia 
provocando que, para cumplir con las normas técnicas vigentes de promulgación de políticas públicas, se deba disminuir 2 puntos porcentuales a la meta original, siendo modificada de la siguiente manera: Incrementar del $27,81 \%$ al $29,21 \%$ la tasa bruta de matrícula en educación superior en Universidades y Escuelas Politécnicas en el año 2021.

\section{Referencias}

Basabe-Serrano, S., Pachano, S., \& Mejía, A. (2010). La democracia inconclusa: Derechos fundamentales, instituciones políticas y rendimientos gubernamentales en Ecuador (1979-2008). Revista de Ciencia Política, 30(1), 65-85, e-ISSN: 0718-090X. Recuperado de: https://doi.org/10.4067/s0718-090x2010000100005

Bravo, O. (2018). Indicadores de gobernanza territorial de los objetivos del Plan Nacional del Buen Vivir (PNBV 2013-2017), Ecuador. Civilizar: Ciencias Sociales y Humanas, 18(35), 25-38, e-ISSN: 2619189X. Recuperado de:

https://doi.org/10.22518/usergioa/jour/ccsh/2018.2/a03

CRE (2008a,b). Constitución de la República del Ecuador. Registro Oficial 449 de 20-oct-2008. Ultima modificación: 13-jul-2011. Quito, Ecuador: Tribunal Supremo Electoral.

Eslava, A. (2011). El juego de las políticas públicas: Reglas y decisiones sociales. ISBN 978-958-720-105-5. Medellín, Colombia: Fondo Editorial Universidad EAFIT.

Jordana, J. (1995). El análisis de los poticy networks: ¿una nueva perspectiva sobre la relación entre políticas públicas y Estado?. GAPP: Gestión y Análisis de Políticas Públicas, (3), 77-89, e-ISSN: 1989-8991. Recuperado de: https://doi.org/10.24965/gapp.vi3.40

LOES $(2018 a, b)$. Ley Orgánica de Educación Superior. Registro Oficial Suplemento 298 de 12-oct.-2010. Ultima modificación: 02-ago.-2018. 
Quito, Ecuador: Asamblea Nacional.

Ostrom, E. (2015). Comprender la Diversidad Institucional. Primera edición electrónica, ISBN: 978-607-16-2733-9. México: Fondo de Cultura Económica.

Pérez, H. (2016a,b). Problemas, actores y decisiones en las políticas públicas. Marco de análisis para el estudio de los programas de crédito educativo en Colombia. Universitas Humanística, (83), 247273, e-ISSN: 2011-2734. Recuperado de:

https://doi.org/10.11144/javeriana.uh83.padp

Ramírez, R. (coord.). (2016a,b,c). Universidad Urgente: para una sociedad emancipada. $1^{\text {a }}$ edición, ISBN 978-9978-339-11-4. Quito, Ecuador: SENESCYT-IESALC.

Ramírez, R., \& Minteguiaga, A. (2010). Transformaciones en la Educación Superior Ecuatoriana: Antecedentes y perspectivas futuras como consecuencias de la nueva constitución política. ESS: Educación Superior y Sociedad, 15(1), 129-154, e-ISSN: 0798-1228. Recuperado de: https://www.iesalc.unesco.org/ess/index.php/ess3/issue/view/40

Rojas, J. (2011). Reforma universitaria en el Ecuador. Etapa de transición. Innovación Educativa, 11(57), 59-67, e-ISSN: 1665-2673. Recuperado de: https://www.redalyc.org/articulo.oa?id=179422350008

Scartascini, C., Spiller, P., Stein, E., Tommasi, M. (ed.). (2011a,b). El juego político en América Latina ¿Cómo se deciden las políticas públicas?. Primera edición, ISBN: 978-958-8307-92-3. Colombia: Mayol Ediciones S.A.

SENESCYT (2020). Matrícula en Universidades y Escuelas Politécnicas.

Versión 2.0. Ecuador: Secretaria de Educación Superior, Ciencia, Tecnología e innovación.

Senplades (2017a,b). Plan Nacional de Desarrollo 2017-2021. Toda una Vida. Quito, Ecuador: Secretaría Nacional de Planificación y Desarrollo. 
Stein, E., Tommasi, M., Echebarría, K., Lora, E., \& Payne, M. (coord.). (2006a,b). La política de las políticas públicas: Progreso económico y social en América Latina. Informe 2006. Primera edición, ISBN: 1-59782-009-1. México: Editorial Planeta Mexicana, S.A. de C.V.

Subirats, J., Knoepfel, P., Larrue, C., \& Varone, F. (2008a,b). Análisis y gestión de políticas públicas. Barcelona, España: Editorial Ariel.

Tamayo, M. (1997). El análisis de las políticas públicas. En Bañón, R., \& Carrillo, E. (comps.). La nueva Administración Pública. (págs. 281-312). ISBN: 84-206-8162-8. Madrid, España: Alianza Editorial, S.A. 


\section{Carlos Andrés Ortega Vega}

e-mail: cortega@senescyt.gob.ec

Nacido en Quito, Ecuador, el 12 de octubre del año 1976.

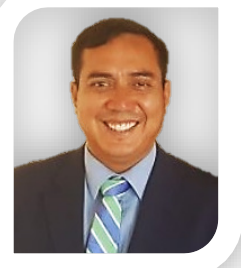

Capitán de Corbeta de la Armada del Ecuador en Servicio Pasivo; Licenciado en Ciencias Navales de Universidad Naval "Comandante Rafael Morán Valverde" (UNINAV); Ingeniero Mecánico de la "Escuela Superior Politécnica del Litoral"; Magister en Educación Superior de la Universidad Casa Grande (UCG); trabajé como Rector y Vicerrector del Instituto Tecnológico Superior "Centro Tecnológico Naval" (CETNAV), durante 6 años; participé en el diseño y rediseño de carreras, acreditación institucional y procesos académicos enmarcados en la Ley Orgánica de Educación Superior (LOES); tengo experiencia docencia universitaria; como militar retirado mantengo solidos principios éticos y morales; actualmente colaboro en con la Secretaría de Educación Superior, Ciencia, Tecnología e Innovación (SENESCYT), donde inicié como Subsecretario de Estado en manejo de institutos tecnológicos públicos y trabajos relacionados a la aplicación de normativas (LOES, RRA, etc.) que rigen a la educación superior; manejo de información del sistema de educación superior dentro de SENESCYT, en conjunto con el Consejo de Educación Superior del Ecuador (CES) y el Consejo de Aseguramiento de la Calidad de la Educación Superior (CACES); y como Coordinador Nacional de Gestión de la Información de la Educación Superior del país. 


\section{Christian Virgilio Guerrero Salazar \\ e-mail: chguerrero.ascaped@gmail.com}

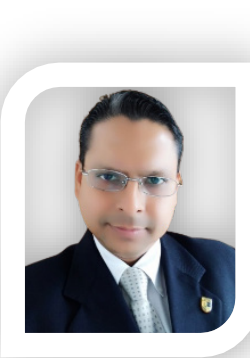

Nacido en Guayaquil, Ecuador, el 8 de julio del año 1977. Licenciado en Ciencias de la Educación, Especialización FIMA de la Facultad de Filosofía, Letras y Ciencias de la Educación de la Universidad de Guayaquil (UG); Maestría en Educación de la Universidad Tecnológica Empresarial de Guayaquil (UTEG); tengo una experiencia docente de más de 20 años colaborando con distintas instituciones de educación media y superior en la ciudad de Guayaquil; soy director pedagógico de Asesoría y Capacitación Pedagógica (ASCAPED), empresa dedicada a la capacitación y asesoría educativa; también me desempeño como docente y gestor pedagógico en la Unidad Educativa Bilingüe Torremar; he colaborado durante varios años con diferentes Universidades, fundaciones y editoriales ecuatorianas como capacitador docente, asesor pedagógico, investigador, itemólogo y metodólogo; soy consultor en el área pedagógica e instructor de cursos de formación de la Organización de Estados Iberoamericanos (OEI); he participado en varios eventos nacionales e internacionales como ponente; cuento con variadas publicaciones en revistas indexadas.

El contenido de este manuscrito se difunde bajo una Licencia de Creative Commons ReconocimientoNoComercial-Compartirlgual 4.0 Internacional 